\title{
A Novel Multiplex Network-based Sensor Information Fusion Model and Its Application to Industrial Multiphase Flow System
}

\author{
Zhong-Ke Gao ${ }^{1}$, Wei-Dong Dang ${ }^{1}$, Chao-Xu Mu${ }^{1}$, Yu-Xuan Yang ${ }^{1}$, Shan $\mathrm{Li}^{1}$, Celso Grebogi ${ }^{2}$ \\ ${ }^{1}$ School of Electrical and Information Engineering, Tianjin University, Tianjin 300072, China \\ ${ }^{2}$ Institute for Complex Systems and Mathematical Biology, King's College, University of Aberdeen, Aberdeen \\ AB24 3UE, UK
}

\begin{abstract}
Increasingly advanced technology allows the monitoring of complex systems from a wide variety of perspectives. But the exploration of such systems from a multi-channel sensor information viewpoint remains a complicated challenge of ongoing interest. As a development of modality transition theory, we first present a novel multiplex network-based model for multichannel sensor information fusion. Toward this aim, projection network and weighted network measures, including average weighted clustering coefficient and graph energy, are exploited both to implement data mining and quantitatively characterize the studied system. In particular, as a validation, the model is tested on spatial-temporal sensor measurements acquired from oil-water flow experiments. The results suggest that our analytical framework allows for the efficient characterization of the spatial flow behaviors underlying the transition of different flow patterns.
\end{abstract}

Index Terms-Signal analysis, Industrial multiphase flow, Multiplex network, Information fusion.

\section{INTRODUCTION}

$\mathbf{W}$ ITH the development of appropriate hardware, multichannel sensor system [1]-[3] has emerged as a key component for providing a comprehensive understanding of complex systems, ranging from physics and chemistry to environment and biology. But the research about how to characterize the intrinsic dynamics underlying the system under consideration is still in its infancy. The measurements from multi-channel sensor system of complex systems are always featured by the strong nonlinearity and coupling characteristics, which makes it difficult to address in a significant way. This calls for intensive research to come up with a novel robust information fusion model and to open up new research venues.

Complex network [4]-[6] theory has undergone an explosive growth in recent years. The substantial and large number of achievements emerging from different fields have demonstrated that complex network can serve as an efficient framework for describing and characterizing complex systems [7]-[16]. Quite recently, complex networks have been obtained and analyzed from time series, and many successful applications have been achieved in different fields [17]-[27]. Ref [28] presents a review of complex network analysis of time series. Moreover, because of the multiplex character of real- world systems, the interest of complex network has gradually changed from single-layer network to multilayer network [29], especially multiplex networks [30]-[32]. Up to now, this burgeoning theory has been applied in systems such as air transportation systems [33], multiplayer online games [34], information spreading [35]-[36] and diffusion process [37].

Oil-water two-phase flow [38]-[39], a primary kind of multiphase flow, occurs in various industrial applications, particularly in the exploitation of oil fields. This mixture flow displays instabilities, transient and random behaviors, and can thus be considered as a typical complex system. Chinese petroleum industry has developed rapidly in recent years and the low velocity oil-water two-phase flow remains in the center of attention for many researchers. Oil phase can disperse in continuous water phase in a variety of temporal-spatial configurations defined as flow patterns [40]-[41]. Different flow patterns exhibit different intrinsic characteristics. Revealing the spatial local flow behaviors of low velocity oil-water flow patterns represents a significant challenge of ongoing interest.

In this paper, based on multiplex network theory, we develop a novel multiplex network-based sensor information fusion model (MSIF model) for exploring multi-channel sensor information. In particular, we obtain the weighted projection network in the MSIF model and then use the average weighted clustering coefficient [42] and graph energy [43] to make a quantitative assessment. By carrying out oil-water twophase flow experiments and applying our MSIF model to the experimental measurements, we find that the complex spatial flow behaviors governing the transitions of different oil-water flow patterns can be effectively characterized.

This paper is organized as follows. Section I gives the introduction of this research. The MSIF model is described in Section II. Section III illustrates the DLDSC Sensor system and oil-water flow experiments. Section IV provides the application of MSIF model to multi-channel oil-water flow measurements. Section V concludes the paper.

\section{MULTIPLEX NETWORK-BASED SENSOR INFORMATION} FUSION MODEL 
I $\mathrm{N}$ this paper, we develop a novel MSIF model to provide an effective framework for analyzing multi-channel sensor information. A schematic diagram of our model is presented in the lower part of Fig. 1. For the double-layer sensor measurements, for example $\left\{x_{k, i}^{u p}\right\}_{i=1}^{N}, k=1,2, \ldots, p$ and $\left\{x_{k, i}^{\text {down }}\right\}_{i=1}^{N}, k=1,2, \ldots, p$, we develop a novel multiplex network, namely modality-based multiplex network (MBMN), with two layers to characterize the studied system. Note that, multiplex network, where the links in different layers denote distinct kinds of interactions between the same set of nodes, is a crucial characteristic of multilayer network.

Based on our previously proposed modality transition-based network theory [24], we perform the following steps to construct the up-layer network $W^{u p}$ of the MBMN from the uplayer signals $\left\{x_{k, i}^{u p}\right\}_{i=1}^{N}, k=1,2, \ldots, p$, where $N$ represents the length of each signal and $p=4$ denotes the number of signals in one of the layers:

(a) Firstly, we divide each channel of the up-layer signals into a multitude of sub-time series with the data length equal to $l$ via a sliding window, where the window slides along time by a step of $\tau$. For each window, we obtain four sub-time series corresponding to the four up-layer signals, respectively.

(b) Then, we calculate the correlation between any two subtime series in the same window by the following equation

$$
r_{x y}=\frac{\sum_{i=1}^{l}\left(x_{i}-\bar{x}\right)\left(y_{i}-\bar{y}\right)}{\sqrt{\sum_{i=1}^{l}\left(x_{i}-\bar{x}\right)^{2}} \sqrt{\sum_{i=1}^{n}\left(y_{i}-\bar{y}\right)^{2}}} .
$$

All six correlation coefficients including $r_{12}, r_{13}, r_{14}, r_{23}$, $r_{24}$, and $r_{34}$ are set as the elements of a modality $r_{12} \rightarrow A, r_{13} \rightarrow B, r_{14} \rightarrow C, r_{23} \rightarrow D, r_{24} \rightarrow E, r_{34} \rightarrow F$.

(c) We rank the six correlation coefficients incrementally to obtain a modality for each sliding window. For instance, if $A<C<B<D<F<E$, then we obtain the modality ACBDFE. The number of all possible modalities is equal to the strings of the permutations of $A, B, C, D, E$ and $F$, i.e., $6 !=720$.

(d) We define each modality as a node and set all the nodes in a fixed order. Then the directed and weighted connections between the nodes can be determined in terms of the direction and times of the transitions among modalities. For instance, in the current step, we acquire the modality ABCDEF (e.g., node $i$ ), and in the next step, the modality become ABEFCD (e.g., node $j$ ), so we get one directed edge from node $i$ to $j$ and the $w_{i j}^{u p}$ pluses one. Self-transitions are excluded.

For the down-layer signals $\left\{x_{k, i}^{\text {down }}\right\}_{i=1}^{N}, k=1,2, \ldots, p$, we implement the analogous steps to obtain the down-layer network $W^{\text {down }}$ of the MBMN. The order of nodes is the same as the up-layer network. Finally, the above procedure allows us to infer a two-layer multiplex network with 720 nodes in each layer. The directed and weighted links in different layers reflect different transition processes of the modalities, which render our method particularly useful for uncovering complicated system behaviors.
Then we derive a weighted projection network $W^{u-d}$ from the inferred MBMN, described as follows

$$
W^{u-d}=W^{u p}+W^{\text {down }},
$$

where $W^{u p}$ and $W^{\text {down }}$ are the directed and weighted matrices of up-layer network and down-layer network, respectively. In Fig. 1, we present a schematic diagram of the simplified MBMN with 10 nodes in each layer and the corresponding directed weighted projection network derived from this multiplex network. The node with identical number in the up-layer network and down-layer network corresponds to the same modality. The structural skeleton of the network is drawn by the software MuxViz [44].

Based on the inferred directed weighted projection network, we calculate the average weighted clustering coefficient and the graph energy to give a quantitatively characterization of the dynamics. The weighted clustering coefficient [42] of node $i$ is defined as follows

$$
C_{i}^{u-d}=\frac{\sum_{j, k} w_{i j}^{u-d} w_{j k}^{u-d} w_{k i}^{u-d}}{\sum_{j, k} w_{i j}^{u-d} w_{k i}^{u-d}}(j \neq k),
$$

where $w_{i j}^{u-d}$ represents the weight of the edge from node $i$ to node $j$ in the weighted projection network $W^{u-d}$. The average weighted clustering coefficient for the $W^{u-d}$ can be calculated by

$$
\left\langle C_{W^{u-d}}\right\rangle=\frac{1}{N} \sum_{i=1}^{N} C_{i}^{u-d} .
$$

The graph energy [43] for a weighted projection network $W^{u-d}$ is the sum of the absolute value of the whole eigenvalues of the network

$$
E_{W^{u-d}}=\sum_{i=1}^{N}\left|\lambda_{i}\right|
$$

where $\lambda_{i}$ denotes the $i$-th eigenvalue of the network $W^{u-d}$ and $\left|\lambda_{i}\right|$ means the absolute value of $\lambda_{i}$.

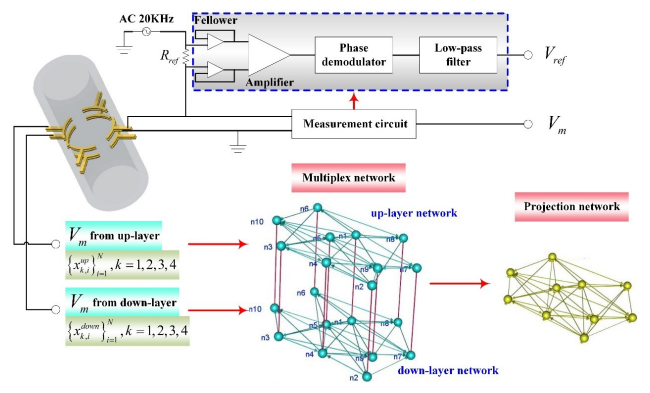

Fig. 1. (Color online) Schematic of multiplex network-based sensor information fusion model. 


\section{Multi-Channel Sensor MEASUREMENT System} AND MULTIPHASE FLOW EXPERIMENTS

\section{A. Measuring circuit for the DLDSC Sensor measurement system}

As an extension of the four-sector distributed conductance sensor [39], we technically design a double-layer distributedsector conductance sensor (DLDSC Sensor), as shown in Fig. 1. In the upper zone of Fig. 1 we give a schematic diagram of the voltage measurement circuit for one sector in the sensor. The other sectors are the same. We obtain the voltage $V_{m}$ following the real-time variation of the multiphase flow in the relative sector of the pipe. The voltage $V_{\text {ref }}$ across a fixed resistance is captured as a reference to calibrate $V_{m}$. Note that, the measurement circuit consists mainly of the voltage follower, amplifier, phase demodulator and low-pass filter. The integrated circuit AD630 is selected as the phase sensitive demodulator. The cut-off frequency of the low-pass filter circuit is set as $30 \mathrm{~Hz}$. In particular, the $20 \mathrm{kHz}$ sinusoidal exciting source is utilized in our voltage measurement circuit. The data acquisition equipment is the National Instrument Corporations data acquisition card PXI 4472 (with synchronized acquiring function and eight sampling channels). The data processing part is realized under LabVIEW operating environments, which allows realizing real-time data waveform displaying, storing and analyzing.

\section{B. Experiments and data acquisition}

We systematically carry out oil-water two-phase flow experiments in a vertical 20-mm-diameter pipe at Tianjin University. The flow loop facility includes a water tank, an oil tank, two peristaltic metering pumps, a mixing tank, a vertical testing pipe, some hand ball valves and our DLDSC Sensor, as shown in Fig. 2. The experimental media are tap-water and white oil with a density of $856 \mathrm{~kg} / \mathrm{m}^{3}$ and a viscosity of $11.984 \mathrm{mPas}$. The DLDSC Sensor consists of two identical parts, namely, up-structure and down-structure sector sensors, which is specially designed for capturing spatial oil-water flow information from different pipe positions. The experimental process can be described as follows: Firstly, the water and oil are pumped into the vertical pipe respectively by two peristaltic metering pumps, which can accurately control the inlet flow parameters of the flow media in light of the pre-defined water-cut and total flow velocity. The oil and water phase first flow through a long vertical pipe until the oil-water flows reach a stable state, namely, until the water and oil adequately mix and a steady flow pattern occurs, and then the double-layer multi-channel experimental information of oil-water flows are acquired via the DLDSC Sensor measurement system. The sampling rate is $4 \mathrm{kHz}$ and the sampling duration for each measurement is $60 \mathrm{~s}$. We acquire eight-channel measurements for one specified flow condition in which the up-structure and down-structure sectors of the DLDSC Sensor get four channels each. Then we change the water-cut and the total flow velocity, and repeat the above operation to generate a new flow condition and acquire again the experimental data. This process lasts until all the flow conditions are fulfilled. In this experiment, the total flow velocity is in the range of $0.0184 \mathrm{~m} / \mathrm{s}-0.2579 \mathrm{~m} / \mathrm{s}$ and the water-cut is set as 70\%, 80\%, 84\%, 94\%, and 98\%, respectively. Meanwhile, the high-speed video camera is used to help classify different experimental flow patterns. During the experiments, three typical water continuous flow patterns are observed, i.e., oil-in-water slug flow (D OS/W), oil-inwater bubble flow (D O/W) and oil-in-water VFD flow (VFD $\mathrm{O} / \mathrm{W})$.

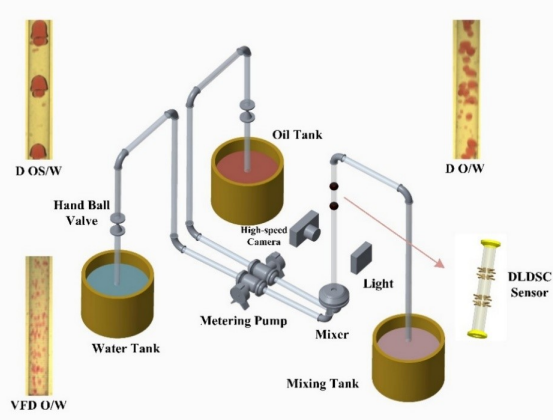

Fig. 2. (Color online) Schematic of vertical upward oil-water two-phase flow loop facility and DLDSC Sensor.

\section{CHARACTERIZING SPATIAL OIL-WATER FLOW BEHAVIORS VIA MSIF MODEL}

A $\mathrm{S}$ a common type of multiphase flow, oil-water flows present strong nonlinear dynamics. Regrettably, despite the tremendous amount of work done on oil-water flows, the complicated flow mechanism underlying low velocity oilwater flows is still elusive. In this section, we introduce the MSIF model and apply it to the fusion of multi-channel DLDSC Sensor measurements. In concrete terms, we derive MBMN and projection network for each flow condition. And subsequently two weighted network measures are extracted to give the quantitatively characterization. All the calculated results are shown in Figs. 3-7, where $K_{w}$ denotes the watercut and $V_{m}$ represents the total flow velocity. As can be seen, for each fixed water-cut, the average weighted clustering coefficient and graph energy exhibit obviously different values for different total flow velocities. They present monotone increasing trends, which allow characterizing the evolution of spatial complicated flow behaviors and provide an intuitively and quantitatively evidence about the power of our model.

In concrete terms, when the water-cut $\left(K_{w}\right)$ is not very high, such as $K_{w}=70 \%$, oil-water flows gradually evolve from oil-in-water slug flow to oil-in-water bubble flow with the increase of total flow velocity $\left(V_{m}\right)$. When the $V_{m}$ is low, the oil phase of the mixture flow gathers into cap-shaped big oil slugs and slithers slowly from the bottom up, namely, the oil-water flows appear as vertical oil-in-water slug flow (D OS/W). Correspondingly, the network measures shown in Figs. 3-5 exhibit small values for such flow pattern, which exactly serve as a good indicator for the quasi-periodic flow behavior and heterogeneous flow feature in such flow pattern. In addition, the increasing trend of the measures demonstrates that the coalescence and slippage between the oil and water 
become weaker with the increase of $V_{m}$, i.e., the small scale oil slugs and oil droplets become more difficult to polymerize into large oil slugs. With the continuous increase of $V_{m}$, the turbulence kinetic energy of the mixture flow is enhanced. When the velocity reaches a critical value, the oil slugs die away and the oil-in-water bubble flow (D O/W) occurs. The oil phase exists over a wide range of scales, randomly flowing up in the water continuum. This transition mainly results from the fact that the oil slugs are broken into oil bubbles induced by the increased $V_{m}$. When mapping these features into the relevant multiplex networks, the network measures of the projection networks maintain the rising trend, suggesting that the spatial heterogeneous distribution is gradually weakened and the spatial local flow behaviors start exhibiting stochastic feature. In particular, the increasing trend of the measures allows distinguishing spatial oil-water flow structures with different form and size, particularly for oil slugs and bubbles under consideration. When the water-cut $\left(K_{w}\right)$ and total flow velocity $\left(V_{m}\right)$ are high, e.g., the $K_{w}$ exceeds $94 \%$ and the $V_{m}$ overs $0.2210 \mathrm{~m} / \mathrm{s}$, the oil bubbles are broken into very fine oil droplets with almost uniform smaller diameters. Meanwhile, the flow patterns evolve from oil-in-water bubble flow (D $\mathrm{O} / \mathrm{W}$ ) to oil-in-water VFD flow (VFD O/W). Correspondingly, the network measures derived from these two flow patterns, as shown in Figs. 6-7, display an increasing trend and their values reach the peak at VFD O/W flow, indicating that the VFD O/W flow presents a more random spatial local flow behavior and a homogeneous spatial distribution of oil droplets.

These interesting results suggest that the average weighted clustering coefficient and the graph energy of the inferred weighted projection networks allow the characterization of the spatial flow behaviors associated with the change of the total flow velocity and water-cut. In summary, the rise of the network measures happens after the change of the spatial local flow behaviors from heterogeneity to homogeneity, which corresponds to the flow transition from D OS/W flow pattern to $\mathrm{D} \mathrm{O} / \mathrm{W}$ flow pattern and then to $\mathrm{VFD} \mathrm{O} / \mathrm{W}$ flow pattern. In particular, the network measures can efficiently reflect the distinct local flow structures associated with the form and size of oil slugs and oil bubbles. These findings render our MSIF model particularly efficient to characterize spatial local flow behaviors of the low velocity oil-water two-phase flow in vertical pipes.

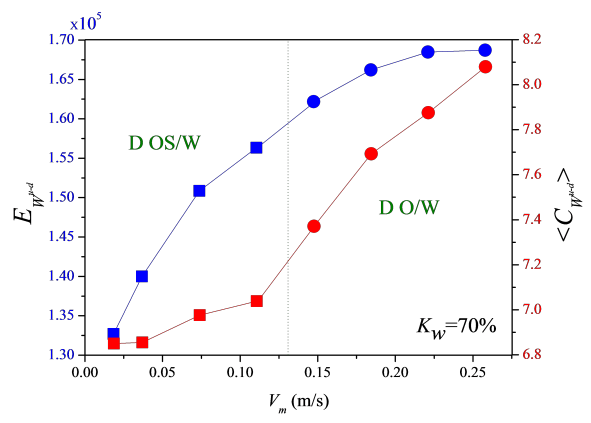

Fig. 3. (Color online) Distribution of the network measures with the change of total flow velocity for different flow conditions when $K_{w}=70 \%$. (a) Graph energy $E_{W^{u-d}}$ (b) Average weighted clustering coefficient $\left\langle C_{W^{u-d}}\right\rangle$.

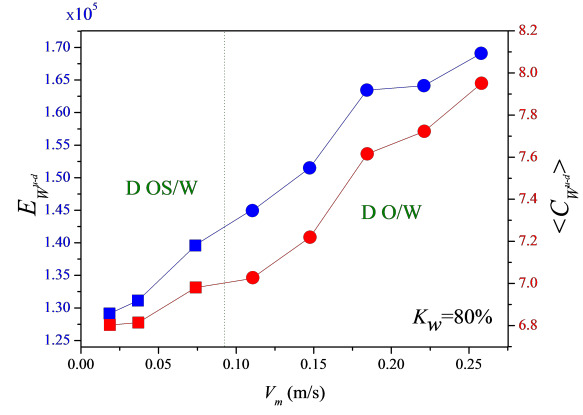

Fig. 4. (Color online) Distribution of the network measures with the change of total flow velocity for different flow conditions when $K_{w}=80 \%$. (a) Graph energy $E_{W^{u-d}}$ (b) Average weighted clustering coefficient $\left\langle C_{W^{u-d}}\right\rangle$.

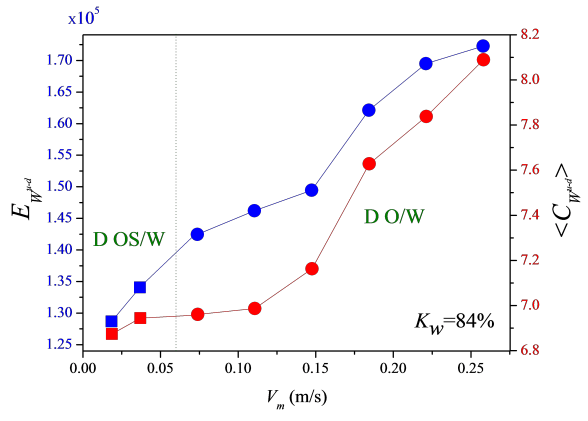

Fig. 5. (Color online) Distribution of the network measures with the change of total flow velocity for different flow conditions when $K_{w}=84 \%$. (a) Graph energy $E_{W^{u-d}}$ (b) Average weighted clustering coefficient $\left\langle C_{W^{u-d}}\right\rangle$.

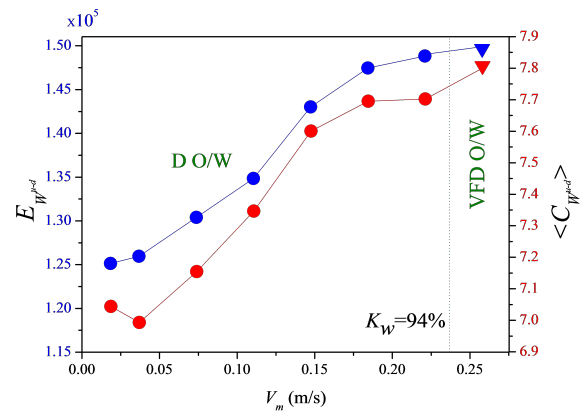

Fig. 6. (Color online) Distribution of the network measures with the change of total flow velocity for different flow conditions when $K_{w}=94 \%$. (a) Graph energy $E_{W^{u-d}}$ (b) Average weighted clustering coefficient $\left\langle C_{W^{u-d}}\right\rangle$. 


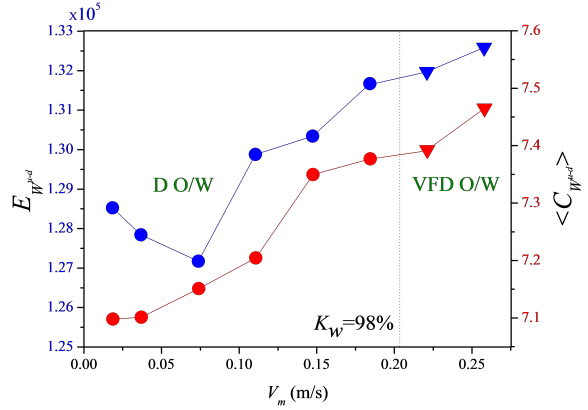

Fig. 7. (Color online) Distribution of the network measures with the change of total flow velocity for different flow conditions when $K_{w}=98 \%$. (a) Graph energy $E_{W^{u-d}}$ (b) Average weighted clustering coefficient $\left\langle C_{W^{u-d}}\right\rangle$.

\section{CONCLuSion}

Fusing and analyzing multi-channel sensor information has unquestionably become a significant procedure for the comprehensive characterization of complex systems. Here a novel MSIF model is established aiming at providing an effective framework for exploring multi-channel sensor information. We first infer MBMN from double-layer multi-channel sensor measurements. Then projection network and weighted network measures, including average weighted clustering coefficient and graph energy, are exploited both to characterize the studied systems. Especially, by applying our MSIF model to experimental DLDSC Sensor measurements of vertical oilwater two-phase flow, we find that the network measures are sensitive to the change of local flow structures and particularly allow the characterization of spatial local flow behaviors of low velocity oil-water flows. Our findings suggest that our MSIF model enables to efficiently fuse multilayer multichannel signals for characterizing the complicated dynamical behaviors underlying time dependent complex system. Taking into account the generality of our MSIF model for information fusion, in the future research, we will attempt to expand and apply this model to other fields, such as brain network analysis and industrial big data mining.

\section{ACKNOWLEDGMENT}

This work was supported by National Natural Science Foundation of China under Grant No. 61473203, and the Natural Science Foundation of Tianjin, China under Grant No. 16JCYBJC18200.

\section{REFERENCES}

[1] P. Y. Chen, S. Yang, and J. McCann, Distributed real-time anomaly detection in networked industrial sensing systems, IEEE Trans. Ind. Electron., vol. 62, no. 6, pp. 3832-3842, Jun. 2015.

[2] F. Dobslaw, T. Zhang, and M. Gidlund, End-to-end reliability-aware scheduling for wireless sensor networks, IEEE Trans. Ind. Informat., vol. 12, no. 3, pp. 758-767, Apr. 2016

[3] H. J. Zhang, H. Xing, J. L. Cheng, A. Nallanathan, and V. C. M. Leung, Secure resource allocation for OFDMA two-way relay wireless sensor networks without and with cooperative jamming, IEEE Trans. Ind. Informat., vol. 12, no. 5, pp. 1714-1725, Oct. 2016.

[4] X. F. Wang and G. R. Chen, Complex networks: Small-world, scale-free and beyond, IEEE Circuits Syst. Mag., vol. 3, no. 1, pp. 6-20, Sep. 2003.
[5] Y. Tang, H. J. Gao, J. Q. Lu, and J. Kurths, Pinning distributed synchronization of stochastic dynamical networks: a mixed optimization approach, IEEE Trans. Neural Netw. Learn. Syst., vol. 25, no. 10, pp. 1804-1815, Oct. 2014.

[6] J. H. Qin, H. J. Gao, and W. X. Zheng, Exponential synchronization of complex networks of linear systems and nonlinear oscillators: a unified analysis, IEEE Trans. Neural Netw. Learn. Syst., vol. 26, no. 3, pp. 510521, Mar. 2015.

[7] M. E. J. Newman, The structure and function of complex networks, SIAM Rev., vol. 45, no. 2, pp. 167-256, Jun. 2003.

[8] W. X. Wang, R. Yang, Y. C. Lai, V. Kovanis, and C. Grebogi, Predicting catastrophes in nonlinear dynamical systems by compressive sensing, Phys. Rev. Lett., vol. 106, no. 15, pp. 154101-1-154101-4, Apr. 2011.

[9] Z. K. Gao, X. W. Zhang, N. D. Jin, N. Marwan, and J. Kurths, Multivariate recurrence network analysis for characterizing horizontal oil-water twophase flow, Phys. Rev. E, vol. 88, no. 3, pp. 032910-1-032910-12, Sep. 2013.

[10] Z. K. Gao, Y. X. Yang, P. C. Fang, N. D. Jin, C. Y. Xia, and L. D. $\mathrm{Hu}$, Multi-frequency complex network from time series for uncovering oil-water flow structure, Sci. Rep., vol. 5, no. 8222, pp. 1-7, Feb. 2015.

[11] W. Zou, D. V. Senthilkumar, R. Nagao, I. Z. Kiss, Y. Tang, A. Koseska, J. Q. Duan, Andrew, and J. Kurths, Restoration of rhythmicity in diffusively coupled dynamical networks, Nat. Commun., vol. 6, no. 7709, pp. 1-9, Jul. 2015.

[12] Z. Wang, S. Kokubo, M. and J. Tanimoto, Universal scaling for the dilemma strength in evolutionary games, Phys. Life Rev., vol. 14, pp. 1-30, Sep. 2015.

[13] W. X. Wang, Y. C. Lai, and C. Grebogi, Data based identification and prediction of nonlinear and complex dynamical systems, Phys. Rep., vol. 644, pp. 1-76, Jul. 2016

[14] L. Z. Wang, R. Q. Su, Z. G. Huang, X. Wang, W. X. Wang, C. Grebogi, and Y. C. Lai, A geometrical approach to control and controllability of nonlinear dynamical networks, Nat. Commun., vol. 7, no. 11323, pp. 1-11, Apr. 2016.

[15] A. Dwivedi, and X. H. Yu, A maximum-flow-based complex network approach for power system vulnerability analysis, IEEE Trans. Ind Informat., vol. 9, no. 1, pp. 81C88, Feb. 2013.

[16] X. H. Yu, and Y. S. Xue, Smart grids: a cyber-physical systems perspective, Proceedings of the IEEE, vol. 104, no. 5, pp. 1058-1070, May. 2016.

[17] J. Zhang, and M. Small, Complex Network from Pseudoperiodic Time Series: Topology versus Dynamics, Phys. Rev. Lett., vol. 96, no. 23, pp. 238701-1-238701-4, Jun. 2006.

[18] L. Lacasa, B. Luque, F. Ballesteros, J. Luque, and J. C. Nuno, From time series to complex networks: The visibility graph, Proc. Natl. Acad. Sci., vol. 105, no. 13, pp. 4972-4975, Apr. 2008.

[19] X. K. Xu, J. Zhang, and M. Small, Superfamily phenomena and motifs of networks induced from time series, Proc. Natl. Acad. Sci., vol. 105, no. 50, pp. 19601-19605, Dec. 2008.

[20] N. Marwan, J. F. Donges, Y. Zou, R. V. Donner, and J. Kurths, Complex network approach for recurrence analysis of time series, Phys. Lett. A, vol. 373, no. 46, pp. 4246-4254, Nov. 2009.

[21] J. F. Donges, R. V. Donner, M. H. Trauth, N. Marwan, H. J. Schellnhuber, and J. Kurths, Nonlinear detection of paleoclimate-variability transitions possibly related to human evolution, Proc. Natl. Acad. Sci., vol. 108, no. 51, pp. 20422-20427, Dec. 2011.

[22] Z. K. Gao, and N. D. Jin, A directed weighted complex network for characterizing chaotic dynamics from time series, Nonlinear Anal. RWA, vol. 13, no. 2, pp. 947-952, Apr. 2012.

[23] Y. Zou, M. Small, Z. Liu, and J. Kurths, Complex network approach to characterize the statistical features of the sunspot series, New J. Phys., vol. 16, no. 013051, pp. 1-18, Jan. 2014.

[24] Z. K. Gao, P. C. Fang, M. S. Ding, and N. D. Jin, Multivariate weighted complex network analysis for characterizing nonlinear dynamic behavior in two-phase flow, Exp. Therm. Fluid Sci., vol. 60, pp. 157-164, Jan. 2015.

[25] M. Murugesan, and R. I. Sujith, Combustion noise is scale-free: transition from scale free to order at the onset of thermoacoustic instability, $J$. Fluid Mech., vol. 772, pp. 225-245, Jun. 2015.

[26] [26] Z. K. Gao, Q. Cai, Y. X. Yang, N. Dong, and S. S. Zhang, Visibility graph from adaptive optimal kernel time-frequency representation for classification of epileptiform EEG, Int. J. Neur. Syst., vol. 27, no. 4, pp. 1750005-1-1750005-12, Nov. 2016.

[27] Z. K. Gao, W. D. Dang, L. Xue, and S.S. Zhang, Directed weighted network structure analysis of complex impedance measurements for characterizing oil-in-water bubbly flow, Chaos., vol. 27, no. 3, pp. 035805-1035805-7, Feb. 2017 
[28] Z. K. Gao, M. Small, and J. Kurths, Complex network analysis of time series, EPL., vol. 116, no. 5, pp. 50001-1-50001-7, Dec. 2016.

[29] S. Boccaletti, G. Bianconi, R. Criado, C. I. Del Genio, J. GomezGardenes, M. Romance, I. Sendina-Nadal, Z. Wang, and M. Zanin, The structure and dynamics of multilayer networks, Phys. Rep., vol. 544, no. 1, pp. 1-122, Nov. 2014.

[30] P. J. Mucha, T. Richardson, K. Macon, M. A. Porter, and J. P. Onnela, Community Structure in Time-Dependent, Multiscale, and Multiplex Networks, Science., vol. 328, no. 5980, pp. 876-878, May. 2010.

[31] L. Sol, M. Romance, R. Criado, J. Flores, A. G. D. Amo, and S. Boccaletti, Eigenvector centrality of nodes in multiplex networks, Chaos. vol. 23, no. 3, pp. 033131-1-033131-10, Sep. 2013.

[32] F. Battiston, V. Nicosia, and V. Latora, Structural measures for multiplex networks, Phys. Rev. E, vol. 89, no. 3, pp. 032804-1- 032804-16, Mar 2014.

[33] A. Cardillo, J. Gomez-Gardenes, M. Zanin, M. Romance, D. Papo, F. D. Pozo, and S. Boccaletti, Emergence of network features from multiplexity, Sci. Rep., vol. 3, no. 1344, pp. 1-6, Feb. 2013.

[34] M. Szell, R. Lambiotte, and S. Thurner, Multirelational organization of large-scale social networks in an online world, Proc. Natl. Acad. Sci. vol. 107, no. 31, pp. 13636-13641, Aug. 2010.

[35] E. Cozzo, R. A. Banos, S. Meloni, and Y. Moreno, Contact-based social contagion in multiplex networks, Phys. Rev. E, vol. 88, no. 5, pp. 0508011-050801-5, Nov. 2013.

[36] B. Min, S. H. Gwak, N. Lee, and K. I. Goh, Layer-switching cost and optimality in information spreading on multiplex networks, Sci. Rep., vol. 6, no. 21392, pp. 1-12, Feb. 2016.

[37] S. Gomez, A. Diaz-Guilera, J. Gomez-Gardenes, C. J. Perez-Vicente, Y. Moreno, and A. Arenas, Diffusion Dynamics on Multiplex Networks, Phys. Rev. Lett., vol. 110, no. 2, pp. 028701-1-028701-5, Jan. 2013.

[38] L. Liu, O. K. Matar, E. S. P. D. Ortiz, and G. F. Hewitt, Experimental investigation of phase inversion in a stirred vessel using LIF, Chem. Eng. Sci., vol. 60, no. 1, pp. 85-94, Jan. 2005.

[39] Z. K. Gao, Y. X. Yang, L. S. Zhai, N. D. Jin, and G. R. Chen, A Four-Sector Conductance Method for Measuring and Characterizing LowVelocity Oil-Water Two-Phase Flows, IEEE Trans. Instrum. Meas., vol. 65, no. 7, pp. 1690-1697, Mar. 2016.

[40] G. W. Govier, G. A. Sullivan, and R. K. Wood, The upward vertical flow of oil-water mixtures, Can. J. Chem. Eng., vol. 39, no. 2, pp.67-75, Apr. 1961.

[41] J. G. Flores, X. T. Chen, C. Sarica, and J. P. Brill, Characterization of oil-water flow patterns in vertical and deviated wells, SPE Prod. Facil., vol. 14, no. 2, pp. 102-109, May. 1999.

[42] I. E. Antoniou, and E. T. Tsompa, Statistical analysis of weighted networks, Discrete dynamics in Nature and Society, vol. 2008, no. 375452, pp. 1-16, Feb. 2008

[43] R. Balakrishnan, The energy of a graph, Linear Algebra Appl., vol. 387, pp. 287-295, Feb. 2004

[44] M. D. Domenico, M. A. Porter, and A. Arenas, MuxViz: a tool for multilayer analysis and visualization of network, Journal of Complex Networks, vol. 3, no. 2, pp. 159-176, Oct. 2014.

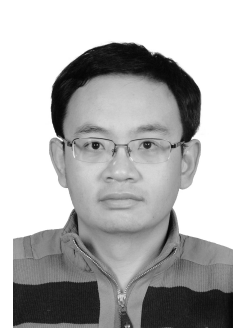

Zhongke Gao received the M.Sc. and Ph.D. degrees from Tianjin University, Tianjin, China, in 2007 and 2010, respectively.

He has been a Professor with Tianjin University since 2016. His current research interests include sensor design, multi-source information fusion, measurement science and technology, big data analysis, and complex networks.

Prof. Gao has been serving as an Editorial Board Member of Scientific Reports and Academic Editor of Plos One since 2014, an Associate Editor of Neural Processing Letters and Royal Society Open Science since 2016, an Associate Editor of IEEE Access since 2017.

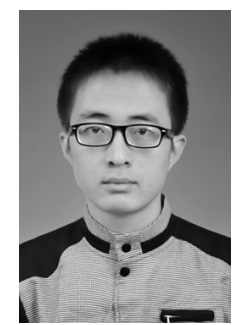

Weidong Dang is currently pursuing the M.Sc. degree with the School of Electrical and Information Engineering, Tianjin University, Tianjin, China.

His current research interests include sensor design, multi-source information fusion, measurement science and technology, multiphase flow, and complex networks.

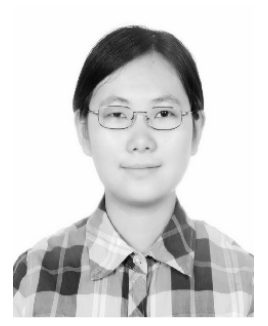

ChaoXu Mu received the Ph.D. degree from Southeast University, Nanjing, China, in 2012.

She has been an Associate Professor with Tianjin University since 2016. Her current research interests include nonlinear control theory and intelligent adaptive control.

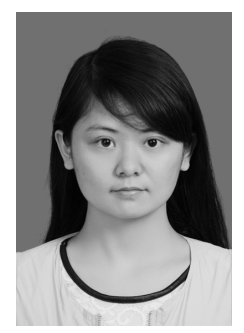

Yuxuan Yang received the M.Sc. degree from Tianjin University, Tianjin, China, in 2017.

Her current research interests include measurement science and technology, multiphase flow, big data analysis, and complex networks.

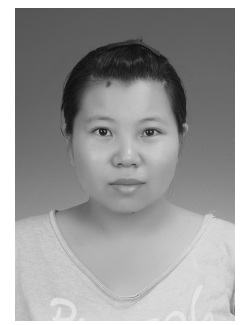

Shan $\mathbf{L i}$ is currently pursuing the M.Sc. degree with the School of Electrical and Information Engineering, Tianjin University, Tianjin, China.

Her current research interests include sensor design, measurement science and technology, multiphase flow, and complex networks. 
Celso Grebogi was born in Curitiba, Brazil, in 1947. He received the B.S. degree from Universi-

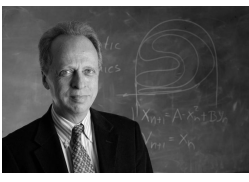

dade Federal do Parana, Parana, Brazil, in 1970, the M.S. degree from the University of Maryland, College Park, in 1975, and the Ph.D. degree from the University of Maryland in 1978.

In 2005, Dr. Grebogi was invited to join the University of Aberdeen as the "Sixth Century Chair in Nonlinear and Complex Systems". There he has been the Founding Director of the Institute for Complex Systems and Mathematical Biology in 2009. He was also the Co-founder of the Institute of Pure and Applied Mathematics in 2013.

His current research focuses on systems biology, neurodynamics, methods to control chaos, the dynamics of spatio-temporal systems, active processes in chaotic flows, relativistic quantum dynamical systems, and nanosystems including graphene and opto-mechanical systems.

$\mathrm{He}$ is a Fellow of The Royal Society of Edinburgh, Fellow of the Brazilian Academy of Sciences, Fellow of The World Academy of Sciences (TWAS/UNESCO), Fellow of the American Physical Society, and Fellow of the Institute of Physics (UK). As one of the participants, he proposed the famous O.G.Y. method for chaos control. And he was listed in the 2016 Thomson Reuters Citation Laureates. 